\title{
Utilizing big data in tourism marketing
}

\author{
Xin $\mathrm{Ye}^{1, \text { a }}$, Wen $\mathrm{Liu}^{2, b}$, Longgang $\mathrm{Li}^{2, \mathrm{c},{ }^{*} \text { and Yaning Zhang }}$, d \\ ${ }^{1}$ School of Management, Minzu University of China, Beijing 100081, China \\ ${ }^{2}$ Department of Military Economy, Logistics University of PAP, Tianjin 300072, China \\ astarnighty@outlook.com, bliuwen522@126.com, ‘410641036@qq.com, dzhangyaning@163.com
}

Keywords: Big data; persona; tourism marketing.

\begin{abstract}
More and more people rely on mobile internet devices, they generate a huge amount of data every day. Tourism market also benefit from the use of big data to make tourist persona and identify demands. The article sketches the development situation and problems of big data application in China, and then give a discussion on big data, tourist persona and precise marketing within related research findings. Others applications and some side effects are also referred.
\end{abstract}

\section{Introduction}

IT industry in China has developed rapidly. With the extensive use of mobile internet, people generate huge amounts of data via mobile phone and other intelligent electronic devices in everyday life. At first sight, these data are scattered messy, while it will become new digital resources if we collect and manipulate them consciously. Different from the traditional statistical one, the big data contain text, voice, images and other forms of unstructured data, which are unable to capture, analyze and manage through the current digital tools at present [1], therefore the study on the effective use of big data is a popular topic.

The market pays more attention to the application of big data in commerce with regard to the scale and status quo of China's big data market. Big data technology will alter the traditional marketing mode and make the business more intelligent as a broader usage scenario. For tourism industry, both online and traditional enterprises mainly make use of big data at the tourism market segmentation, customer market positioning and tourism marketing decision-making process. Offline travel agencies, traffic sectors and other tourist service providers will submit operational data to the big data solution facilitators, meanwhile the tourist attractions or tour destinations will benefit from the analysis reports. For online service, online travel agencies (OTA) collect user information to integrate travelers' schedule, reservation and other data through the platform, then depict detailed tourist persona. With the collection of accurate user generate content (UGC), keyword search index and other data sources, big data can be a new analysis tool to take advantage of a large-scale data compared with traditional sample survey or interview method. It's needed to be further explored that how to filter redundant data, cope with a large amount of unstructured data, and acquire the big data resources on purpose.

\section{Current Problem}

For big data resources, the freshmen concern about the way data are collected. For example, questions like "How to promote innovation in sustainable tourism marketing in the age of big data", "How to achieve precise tourism marketing with the help of big data technology?" are raised. Professionals prefer to place emphasis on collecting big data for accurate visitor persona, such as "Compared to the changes in tourism information, what does the big data make a difference for tourist persona, data reliability and other issues, to guide the industry's cutting-edge direction". The tourist persona is clearly portrayed by big data, and then identify the perceived tourists' characteristics accurately in precision marketing. Many tourism destinations are facing the limited resources, which hampers sustainable development. For that reason, resources will be preferentially assigned to the target area to improve the rate of return on investment. In view of the realistic tourism operation, there are problems in the use of tourism flow data, intended to rationally control the tourists flow. 
The regulation and management is intended to swarm into one place at the same time to ensure the quality of tourists' experience and promote long-term development of tourism destination. The public officer suggest tourism industry should keep developing at a fast pact, the efficiency itself is important as stakeholders want to benefit from the investment quickly, however some experience indicate haste makes waste in my opinion, rushing forward may violate the purpose of tourism management.

\section{Literature Review}

In recent years, technologies like Internet, internet of things (IoT), and cloud computing develop rapidly. The usage scenario is constantly enriched, and massive digital information are generated by kinds of users every day. These data with large capacity can be quickly retrieved, and they are classified as social network text, audio \& video and smart phone GPS positioning data et al [2]. The world's major countries attach great importance to these new resources in the field of big data, the EU issued "Open Data Strategy" to guide big data application in December 2011, the United States launched the "Big Data Research and Development Strategy" in 2012. Senior government authority in China, which owns the most netizens, pay attention to the strategic significance of big data development as well.

\subsection{Big Data and Tourism Industry}

There are experts have discussed issues about the concept of tourism information for many years, with the concept of smart tourism and big data in recent years, they combine these things together to analyze one more step. Zhang Lingyun thought that the smart tourism was based on a new generation of information and communication technology to meet the individual needs of tourists to achieve the effective use of tourism resources and change social resources [3]. The basic plan is to optimize the tourism activities, enhance the visitor experience, and help the tourism enterprises and industry management officers to optimize tourism management, tourism services and wisdom marketing, thus the main goal is met that advance intellectual tourism activities [4]. For China's tourism industry, smart tourism is also a new orientation to apply for tourism destinations, enterprises and tourists themselves [5]. Li Yunpeng (2014) correlated tourism information and smart tourism, he noted the meaning of smart tourism should be based on a new generation of information technology and combined the original technology with the traditional service industry for modern service and industry structure upgrades, this structure upgrade [6]. The most basic value is the tourism information service.

A new generation of information technology reflects the mobility; ubiquitous mobile network popularization is the smart tourism to implement the technical thrust. With the help of smart phones, wearable equipment and other portable electronic tools, tourists can get access to network at anytime, anywhere, thus the usage scenarios can be expanded. Countless real-time online user generated contents are upload and download every day, the users are contributors for big data and also beneficiaries. Liang Changyong, Ma Yinchao and Lu Caihong thought that big data mining is the core of smart tourism, it could embody value of big data for tourism development only if we fully utilized the potential information on tourism big data, and provided comprehensive quality services with considerate decision-making management [7].

\subsection{Big Data and Tourist Persona}

Big data is becoming a new factor of production after the land, labor and capital, it can be the core competence of one enterprise for future development. The firms integrate internal data to promote the sharing of resources between business segments and new product form, meanwhile they build open platform to solicit external partners getting involved in business, and such as the hotel and ticket reserving agents supported by Ctrip or others OTAs. They can make use of big data at larger scale in order to improve enterprise's innovation and overall competency [3]. Many companies keep investing in big data research and development to innovate for this purpose.

Before the Mobile Internet age, some scholars have researched perception of tourists and image of tour destinations through the user generated network text [8], they use "keyword" from Baidu engine to find eligible pages, blogs and comments to combine online text data with traditional qualitative method. Hidden information under network text such as valuable traveling routines, potential travel 
clients, and tour destination recommendations are mined [1]. Since mobile intelligent devices were widely used, people are more likely to share and use data all the time.

According to big data resources, target customers are identified accurately, and then tourist persona can also be acquired. "Persona" is known as multi-dimensional descriptive unstructured data, which record preferences, habits and attitudes of consumers implicitly or explicitly, originated from their online browsing or comments. The tourists belong to consumers, and the tourist persona is depicted through some steps from the collected data. Firstly, the data collected by the terminal equipment are classified into generalized and specified data of the tourists, and then the data are cleaned, de-noised, and normalized. The data are deeply excavated by multivariate analysis, spatial statistics and machine mining, interactive verification between the various types of data, finally they get more accurate results for visual tourist persona processing.

\subsection{Big Data and Travel Precision Marketing}

The application of big data in the field of tourism changes the behavior of tourists and tourism enterprises [9]. Based on the data mining analysis, we can obtain effective tourist persona and position target market directly, identify ideal consumer groups accurately. Scholars at home and abroad have ever tried to use the data captured online to recognize tourist groups. Marineroig and Clavé collected over 100,000 English traveling journals and visitors' comments who had visited Barcelona over the past 10 years, using business intelligence (BI) to analyze large-scale user data [10], with data extraction, cleaning, and target the location and brand marketing of the travel destination management information system (DMIS), and analyze the travel destination performance [11]. Önder used the photo sharing site Flickr as a resource to capture photos of Austrian geography, by collecting and refining picture data, depicting the flow of tourists and clustering analysis. The different tourist groups of destination in combination. At such a small scale area, targeting advertisement in large tourist destinations is more meaningful [12]. Tourism is a comprehensive industry including food \& beverage, accommodation, transportation and entertainment. Big data technology breaks the boundary between traditional tourism industry and share tourists' information in related industries more easily [9].

\subsection{Big Data Apply to Tourism Marketing}

The use of big data is to depict tourist persona, accurately position tourism marketing, predict the overall demand for tourists, and optimize the allocation of resources and product development, in order to control the cost increase profits, control transport volume, reduce the residents' discomfort, and eliminate conflicts in a community. It can protect the destination ecological, economic and social sustainable development with the tourism development.

Visitors have a lot of research results, the traditional use of statistical data in time series, neural networks or economic models are used to predict the tourists flow. The introduction of big data methods import model analysis has more rapid and accurate predictive performance. The search engine like Google or Baidu are based on the keyword search for real-time volume and trend, Beyesian FAVAR, Support Vector Regression (SVR), BP Neural Network, vector-independent autoregressive (VAR) and so on are all metrology models proposed by researchers. The mathematical model of measurement is different from the data source due to the different design of each study, and the results of the best prediction model are not usually the same. Li, Pan, Law and Huang captured Baidu and Google search keyword "Beijing tourism" to observe its sound volume trend, the introduction of the generalized dynamic factor model (GDFM) predicted the amount of visitors to visit, and then compared the amount of actual visit [13]. The forecast performance is better than the classic time series and other statistical models. The large number of tourists, the tourism management departments utilize information resource to guide the establishment of all aspects of tourism activities to cover the operation of the big data monitoring platform to control the tourists flow, and regulate the tourist facilities are evenly distributed in the tourist path to promote the development of tourism destinations and balance in time and space [9]. 


\section{Case Study}

People think intuitively the heater that search for a scenic spot keyword, the more concerned about the attractions where people surge into. Baidu is China's top search engine, with a huge number of tourism search data in depth, can be found in tourism-related words search volume and the actual number of trends are closely linked. Baidu builds a tourism forecasting model, intercepts the daily data of the attention degree of the scenic spots in the search engine, and analyzes the weekly rules of the attention, the seasonal characteristics and the trend of the calendar year [14]. Huang Xiankai, Zhang Lifeng and Ding Yusi took the Baidu search index of Beijing Imperial Palace as an empirical analysis to verify that there is a positive correlation between the trend of network search and the actual tourist quantity of scenic area, which proved the feasibility of predicting the number of tourists through search index [15]. The actual number of visitors to the Forbidden City is provided by the Beijing Municipal Tourism Commission, and it is compared with the forecast value of Baidu's big data department. As shown in Fig. 1, it is found that the forecasting model results are basically the same as the actual trend of the day.

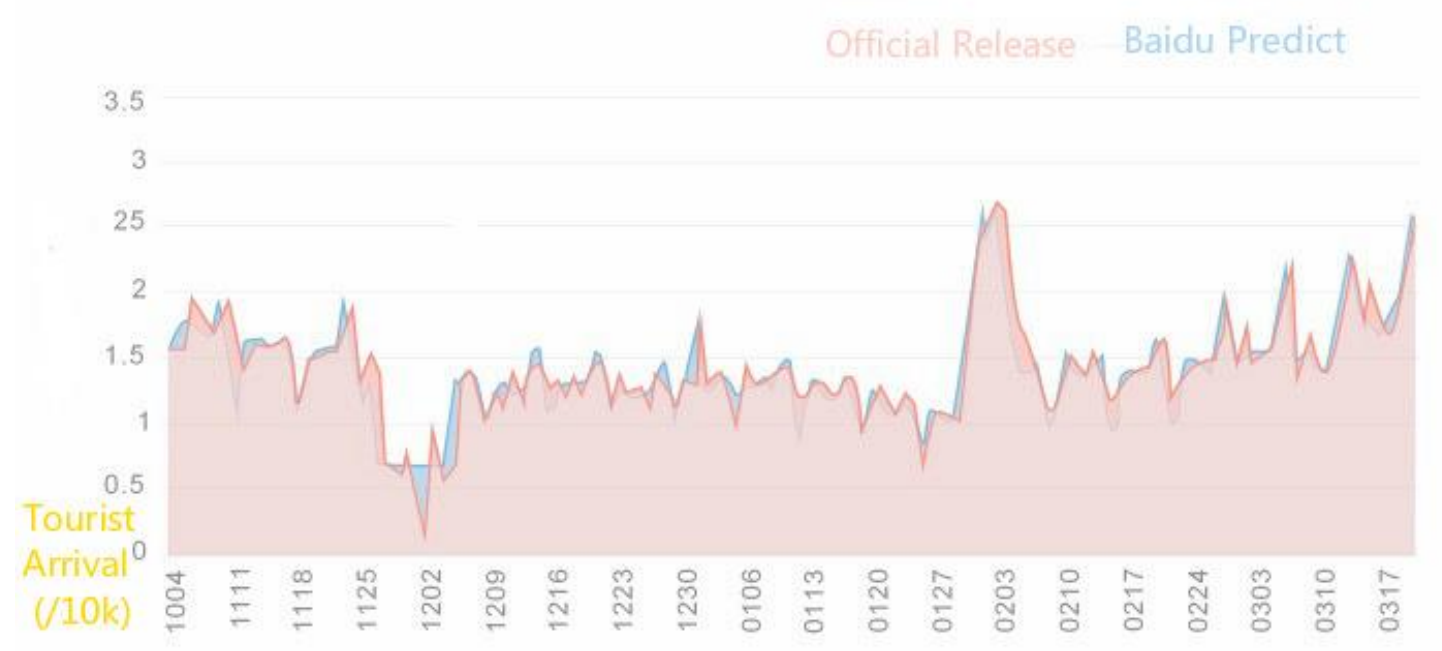

Figure 1. Tourists Arrival of Forbidden City between Official and Baidu

Jiuzhaigou decided to cooperate with Baidu, an outstanding real-time monitoring of traffic and tourist solution provider, to utilize the big data to predict the arrival. According to the use of Baidu products visitors' location, real-time traffic in the scenic site to predict the presentation; according to the user search keywords ahead of the forecast on the day and the next two days, Jiuzhaigou's office are able to facilitate the decision-making before the tourists. At the same time with Baidu forecast results, the managerial department develops different operating solution for different traffic volume to promise safe operation of people and transport facilities. Especially in the small holiday and the Golden Week, the smart controlling system makes effective arrangements and release visitors, in order to enhance the efficiency of scenic spots and visitors' tourism experience.

Baidu in the area of the layout of the golden eye devices. The devices can sense visitors' near field and other mobile phone equipment to obtain the input of tourists through them. Their location information, each person's location aggregated into large amounts of data uploaded to Baidu's cloud analysis platform, together the mobile phones and other equipment App booking information are also spread to the platform. The big data platform of Baidu will get the data to clean, extract and visualize, then we can get predictive analysis results. On one hand, the forecast information through the cloud to the tourists on the mobile phone and other equipment; on the other hand, the real-time data and analysis of the proposed are uploaded to the Jiuzhaigou managerial department. The electronic screens display real-time forecast scenic spot to deliver traffic flow, and the instant information can regulate the choice of travelling route. 


\section{Conclusion and Discussion}

According to the statements above, it takes a brief look at the prospect of utilizing big data in tourism marketing. The process is to make accurate tourist persona, target on audience, optimize the allocation and development of resources, and forecast tourist flow, in order to achieve the sustainable development of tourism. In addition to the previous discussion, some other questions related with big data are ought to be discussed.

For many common tourist attractions, big data can help them notice some patterns uneasy to get in usual life. The classic case "Beer and Diapers" from Wal-Mart is an early successful example using sales data by association algorithm, further the use of big data is more easily to relate something with unexpected reason, it inspires tourism developers to correlate store and tourist attraction, as well as brand and consumer preference.

For academia, the big data tool enables scholars to break the limit. For instance, online UGC is a kind of data easy to be captured, though the format is unstructured, it's feasible to extract a convincing keyword and conduct reasonable network relationship at high quantity level. It indicated the reliability of research method with online data to investigate the perception of tourism image in Xi'an by Zhang Zhenzhen and Li Junyi [16]. They combined the traditional questionnaire survey and the network text data analysis, and two types of measurements aligned fairly well. By the way, the field work is not out-of-date even if the electronic devices have been widely applied. Some researchers realize the online comments mention only a few things about social images of destinations, while they are crucial for researchers and managers, the use of questionnaire and interview will contribute.

At the end, we should note that application of technology may occur some ethical issues about personal privacy. It's fine to obtain public data from blog, Twitter or Weibo as academic resources, while it's quite different to capture information like spider from some private platform such as WeChat Moments, Qzone and personal homepage which are designed for sharing ideas and emotions with close friends only. The smartphone users face the similar problems because it's much easier to detect personal location via GPS in the phone. Whatever good purposes are, just like efficient management and research, it's hard to estimate the negative perception tourists have if they realize they are real-time positioned of GPS by their smart devices. In sum, we must realize that the big data technology need to facilitate the tourists, it's no longer a human technology if the rights of the data generator are offended.

\section{References}

[1]. Xin Guo. Research on tourism big data and mining analysis. Computer Knowledge and Techonology. Vol. 9 (2013) No. 14, p. 3215-3216.

[2]. Mcafee A, Brynjolfsson E. Big data: the management revolution. Harvard Business Review. Vol. 90 (2012) No. 10, p. 60-6, 68, 128.

[3]. Lingyun Zhang. Smart Tourism: Personal customize and the time of public intelligent service. Tourism Tribune.

[4]. Bo Zhou, Lingqiang Zhou. The research on commercial pattern of smart tourism abroad and indication for China. Vol. 31 (2016) No. 6, p. 8-9.

[5]. Li Y, Hu C, Huang C, et al. The concept of smart tourism in the context of tourism information services. Vol. 58 (2017) No. 3, p. 293-300.

[6]. Yunpeng Li, Zhongzhou Hu, Chao Huang, et al. The concept of smart tourism under the context of tourism information service. Vol. 29 (2014) No. 5, p. 106-115.

[7]. Changyong Liang, Yinchao Ma, Caihong Lu. Big data mining: the core of smart tourism. Development Research. Vol. 180 (2015) No. 5, p. 134-139.

[8]. Zhenbin Zhao, Jiao Dang. The travel behavior of backpackers of Mountain Taibai based on internet text content analysis. Vol. 26 (2011) No. 1, p. 134-139.

[9]. Xiaoyun Tang. Grasping macro-control initiative for tourism administration with big data. Tourism Tribune. Vol. 29 (2014) No. 10, p. 9-11. 
[10]. Marineroig E, Clavé S A. Tourism analytics with massive user-generated content: a case study of Barcelona. Journal of Destination Marketing \& Management. Vol. 4 (2015) No. 3, p. 162-172.

[11]. Fuchs M, Höpken W, Lexhagen M. Big data analytics for knowledge generation in tourism destinations: A case from Sweden. Journal of Destination Marketing \& Management. Vol. 3 (2014) No. 4, p. 198-209.

[12]. Önder I. Classifying multi-destination trips in Austria with big data. Tourism Management Perspectives. Vol. 21 (2017), p. 54-58.

[13]. . Li X, Pan B, Law R, et al. Forecasting tourism demand with composite search index. Tourism Management. Vol. 59 (2017), p. 57-66.

[14]. Ying Huang, Zhi Zhou, Juan Huang. Temporal and spatial variations of rural tourism development in age of big data. Acta Agriculture Zhejiangesis. Vol. 26 (2014) No. 6, p. 17091714.

[15]. Xiankai Huang, Lifeng Zhang, Yusi Ding. Study on the predictive and relationship between tourist attractions and the Baidu index: A case study on the Forbidden City. Tourism Tribune. Vol. 28 (2013) No. 11, p. 93-100.

[16]. Zhenzhen Zhang, Junyi Li. A comparative study on the data of a questionnaire and web texts in tourism image researches: A case study of Xi'an's tourism image perception. Tourism Science. Vol. 28 (2014) No. 6, p. 73-81. 